\title{
Performance Evaluation of Bidirectional Optical Amplifiers for Amplified Passive Optical Network Based on Broadband Light Source Seeded Optical Sources
}

\author{
Byoung-Wook Kang and Chul Han Kim* \\ School of Electrical and Computer Engineering, University of Seoul, Seoul 130-743, Korea
}

(Received December 30, 2010 : revised February 28, 2011 : accepted February 28, 2011)

\begin{abstract}
We have evaluated the performances of bidirectional optical amplifiers which were suited for the costeffective implementation of amplified bidirectional passive optical networks (PONs). First, we measured the maximum gains of two simple bidirectional optical amplifiers implemented without using any optical components for the suppression of reflected signals. From the results, the maximum gains of two simple bidirectional amplifiers with a broadband light source (BLS) seeded optical source were limited to be 27 $\mathrm{dB}$ due to the reflection-induced in-band crosstalk, when the reflectance coefficients were measured to be $-33 \mathrm{~dB}$ in both directions. Then, we have also implemented a bidirectional optical amplifier with two band splitters for the amplified bidirectional PON where the two different wavelength bands were allocated to the downstream and upstream signals transmission. In our measurement, we confirmed that the maximum gain of bidirectional optical amplifier with two band splitters could be increased to more than $30 \mathrm{~dB}$ owing to the efficient suppression of in-band crosstalk.
\end{abstract}

Keywords: Passive optical network, Bidirectional optical amplifier

OCIS codes : (060.0060) Fiber optics and optical communications; (060.2330) Fiber optics communications

\section{INTRODUCTION}

Amplified passive optical networks (PONs) based on timedivision multiplexed (TDM) and/or wavelength-division multiplexed (WDM) technologies have been widely demonstrated for the next-generation optical access networks [1-4]. This is because various optical amplifiers, such as erbium-doped fiber amplifier (EDFA), semiconductor optical amplifier (SOA) and Raman amplifier, could provide an extended reach and/or a high split-ratio for the cost-effective implementation of PONs. However, it has been well known that the performance of bidirectional transmission systems over a single fiber could be degraded by the in-band crosstalk [5]. In principle, the in-band crosstalk components would be generated by a double Rayleigh backscattering (DRB) and discrete reflection points within a transmission fiber, and amplified with the gain of bidirectional optical amplifier. In addition, broadband light source (BLS) seeded optical sources, such as a wavelength-locked Fabry-Perot laser diode (FP-LD) and a reflective SOA (RSOA), have been widely used for the cost-effective implementation of hybrid TDM/WDM-PONs or WDM-PONs [6-7], since these sources could provide a colorless operation and a signal modulation/amplification using a single device. Thus, we believe that the maximum gain of bidirectional optical amplifiers should be evaluated properly in order to estimate the maximum reach and splitratio of amplified PONs based on the BLS seeded optical sources.

In this paper, we investigate the effect of reflection-induced in-band crosstalk on the maximum bidirectional gain in an amplified PON using a BLS seeded RSOA source. First, two simple configurations of bidirectional optical amplifier are used for the performance evaluation of amplified PONs. Using two simple bidirectional amplifiers implemented without any optical components for the suppression of reflected signals, we measure the maximum achievable gain of optical amplifier within a negligible in-band crosstalk penalty. Then, we also implement a bidirectional optical amplifier with two band splitters for the efficient suppression of in-band crosstalk in an amplified PON. From the results, we confirm that the bidirectional optical amplifier with two band splitters would be well suited for use in an amplified hybrid TDM/WDMPON and/or WDM-PON with the separated wavelength bands allocated to the downstream and upstream signals transmission.

\footnotetext{
*Corresponding author: chkim@uos.ac.kr

Color versions of one or more of the figures in this paper are available online.
} 


\section{RESULTS AND DISCUSSION}

Figure 1 shows our experimental setup for the performance evaluation of bidirectional optical amplifiers in an amplified PON based on a BLS seeded optical source. A spectrumsliced amplified spontaneous emission (ASE) beam from a BLS was generated and launched into a RSOA via a circulator and an arrayed waveguide grating (AWG). The AWG used in our measurement had a channel spacing of $100 \mathrm{GHz}$ and a 3-dB bandwidth of $0.4 \mathrm{~nm}$. The injected power level of a spectrum-sliced ASE beam into the RSOA was measured to be $-12 \mathrm{dBm}$. A RSOA having threshold current of $13 \mathrm{~mA}$ was used for the amplification and the direct-modulation of the downstream signal. In our measurement, the downstream signal operating at a wavelength of $1550.1 \mathrm{~nm}$ was modulated with a $1.25 \mathrm{~Gb} / \mathrm{s}$ non-return-to-zero (NRZ) pseudorandom pattern of length $2^{31}-1$. The bias current of the RSOA and the data amplitude of the pulse pattern generator (PPG) for the direct-modulation were $24 \mathrm{~mA}$ and $2 \mathrm{~V}_{\mathrm{pp}}$, respectively. Thus, the extinction ratio of our transmitter was measured to be $\sim 11.3 \mathrm{~dB}$. The modulated downstream signal passed through two circulators; one for a BLS seeding into the RSOA and the other for the separation of downstream and upstream signals, and then transmitted through a $25-\mathrm{km}$ single mode fiber (SMF) before being amplified with a bidirectional optical amplifier. Two $2 \times 2$ optical couplers were placed before and after the bidirectional optical amplifier to monitor the amplifier gain with $1 \%$ taps. After passing through another 3-km SMF and a second AWG, the amplified downstream signal was detected with a PIN-based optical receiver (RX) having a $940-\mathrm{MHz}$ electrical filter. In order to simulate the bidirectional signal transmission over a single fiber, an upstream signal of $1550.9 \mathrm{~nm}$ was generated with a spectrum-sliced ASE from another BLS and then transmitted through a 3-km SMF, a bidirectional optical amplifier and a $25-\mathrm{km}$ SMF.

As it can be seen in Fig. 1, two $2 \times 2$ optical tap couplers were used to measure the gain of the bidirectional optical amplifier as well as the reflectance coefficients including both effects of distributed Rayleigh backscattering and discrete reflections within a $25-\mathrm{km}$ and a $3-\mathrm{km}$ SMF links. Fig. 2 shows the optical spectra measured at the $1 \%$ taps of $2 \times 2$ optical couplers placed before and after the bidirectional optical

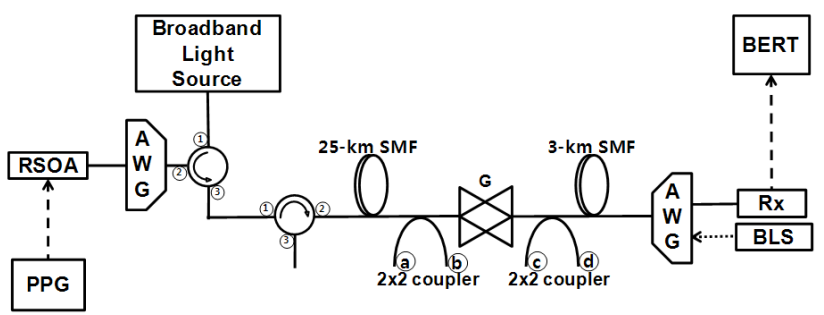

FIG. 1. Experimental setup for the performance evaluation of an amplified PON using a BLS seeded RSOA source and a bidirectional optical amplifier. amplifier. The gain of downstream signals operating at 1550.1 $\mathrm{nm}$ could be estimated from Fig. 2(b) and (d). In addition, the reflectance coefficient from a 3-km SMF could be estimated with the optical spectra of reflected and amplified downstream signals shown in Fig. 2(c) and (d). From the measured optical spectra, the reflectance coefficients were estimated to be $-33 \mathrm{~dB}$ in both $25-\mathrm{km}$ and $3-\mathrm{km}$ SMF links. We also confirmed that these measured reflectance coefficients did not depend on the input power levels of downstream and upstream signals into the bidirectional optical amplifier.

In order to evaluate the maximum gains of bidirectional optical amplifiers in an amplified PON shown in Fig. 1, we first used two simple bidirectional optical amplifiers; one was simply implemented without an isolator as shown in Fig. 3(a), and the other with two circulators and two conventional EDFAs having optical isolators as shown in Fig. 3(b). From the configurations of two simple bidirectional optical amplifiers, it is clear that the in-band crosstalk-to-signal ratio could be estimated with $G^{2} R_{1} R_{2}$ in Fig. 3(a) and $G_{1} G_{2} R_{1} R_{2}$ in Fig. 3(b), where $G, G_{1}$ and $G_{2}$ were the bidirectional gain, the unidirectional gain of downstream signal and upstream signal in the optical amplifiers, and $R_{1}$ and $R_{2}$ were the reflectance coefficients at both sides of bidirectional amplifiers, respectively $[5,8]$. In case of Fig. 3(a), the small amount of reflected $\left(\mathrm{R}_{2}\right)$ downstream signal was amplified $\left(\mathrm{GR}_{2}\right)$ with the upstream signal in a bidirectional optical amplifier. Then, this amplified reflected signal was reflected $\left(\mathrm{R}_{1} \mathrm{GR}_{2}\right)$ and amplified $\left(G^{2} R_{1} R_{2}\right)$ again. Even in the second simple optical amplifier of Fig. 3(b) which was implemented with two unidirectional amplifiers and two circulators for the separate amplification of downstream and upstream signals, the reflected $\left(R_{2}\right)$ downstream signal was amplified $\left(\mathrm{G}_{2} \mathrm{R}_{2}\right)$ with the upstream signal in the lower amplification path, and then reflected $\left(\mathrm{R}_{1} \mathrm{G}_{2} \mathrm{R}_{2}\right)$ and amplified $\left(\mathrm{G}_{1} \mathrm{G}_{2} \mathrm{R}_{1} \mathrm{R}_{2}\right)$ again

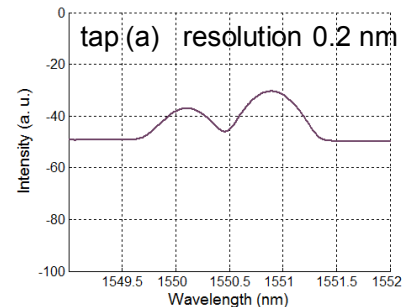

(a)

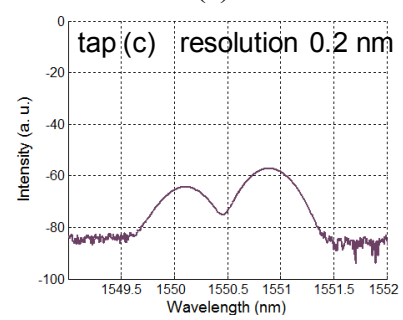

(c)

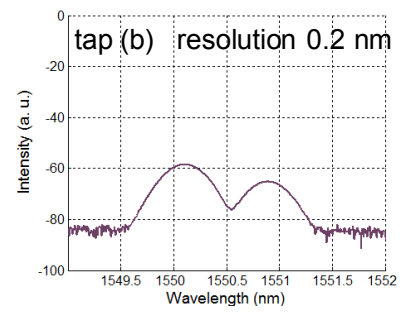

(b)

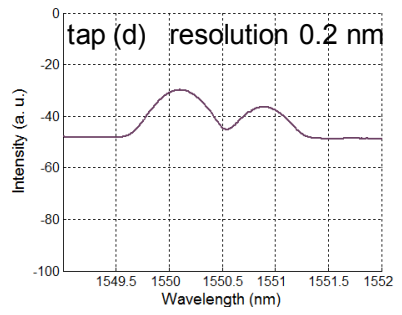

(d)
FIG. 2. Optical spectra of downstream and upstream signals measured at $1 \%$ taps of $2 \times 2$ couplers placed before and after the bidirectional optical amplifier. 

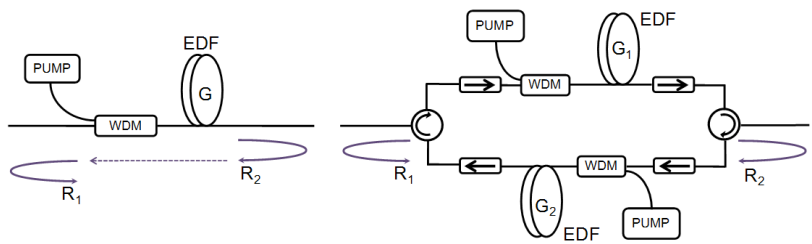

(a)

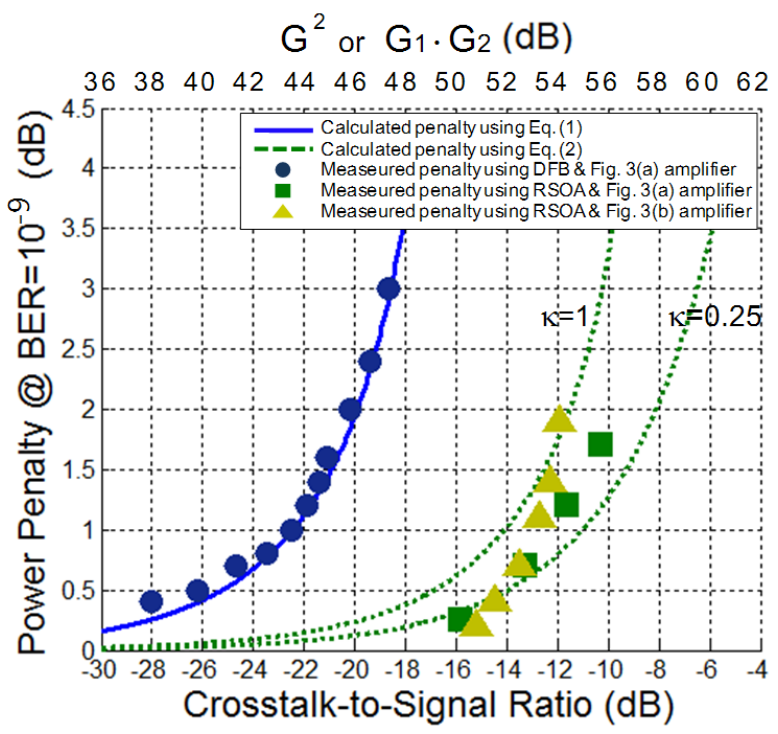

(c)

FIG. 3. Two simple bidirectional optical amplifiers (a) without isolator and (b) with two circulators and unidirectional EDFAs, and (c) measured and calculated in-band crosstalk induced penalties as a function of crosstalk-to-signal ratio with a DFB-LD and a BLS seeded RSOA source.

with the downstream signal in the upper amplification path. Even though the circulator had an isolation of $\sim 30 \mathrm{~dB}$, it could not suppress the reflected and double-reflected signals at all. These double-reflected and amplified components having the same wavelength as the downstream signal were detected simultaneously with the original downstream signal at the optical receiver. Thus, these in-band crosstalk components could not be separated with an optical bandpass filter in the optical receiver and thus they degrade the system's performance. Therefore, we expected that there would be no difference in the in-band crosstalk penalties between two bidirectional optical amplifiers, since we did not use any optical components to suppress the unwanted reflected signals in two separate paths of Fig. 3(b). Fig. 3(c) shows the measured in-band crosstalk penalties as a function of crosstalk-to-signal ratio with two bidirectional optical amplifiers shown in Fig. 3(a) and (b). For all measurements with an amplified PON shown in Fig. 1 , both $R_{1}$ and $R_{2}$ were measured to be $-33 \mathrm{~dB}$. Thus, the crosstalk-to-signal ratio could be changed by simply adjusting the gain of optical amplifiers. As expected, the in-band crosstalk penalties with two bidirectional optical amplifiers were measured to be about the same, as shown in Fig. 3(c). However, with a crosstalk-to-signal ratio of $>-12 \mathrm{~dB}$, the in-band crosstalk penalties with the amplifier of Fig. 3(b) were slightly higher than those with the amplifier of Fig. 3(a). Apparently, the crosstalk-to-signal ratio of $-12 \mathrm{~dB}$ corresponded to the unidirectional amplifier gain of $\sim 27 \mathrm{~dB}$ in Fig. 3(b). Thus, this high amplifier gain with the isolation level $(\sim 30 \mathrm{~dB})$ of a circulator could generate the additional in-band crosstalk components within the amplifier of Fig. 3(b), which in turn would induce the additional penalty. In addition, the in-band crosstalk penalty with two externally-modulated DFB-LDs for downstream and upstream signals was measured and represented in Fig. 3(c), for comparison. From these results, we confirmed that a BLS seeded RSOA source was $\sim 10$ $\mathrm{dB}$ more tolerant to the in-band crosstalk than a DFB-LD source. Thus, for example, using a $1-\mathrm{dB}$ penalty guideline, the maximum bidirectional gain $(\mathrm{G})$ of amplifier shown in Fig. 3(a) could be increased to be $27 \mathrm{~dB}$ with a BLS seeded RSOA source and a reflectance coefficient of -33 $\mathrm{dB}$ in both directions, while the bidirectional gain with a DFB-LD was limited to be $\sim 22 \mathrm{~dB}$.

The in-band crosstalk induced penalties with a DFB-LD [8] and a BLS seeded optical source [9] in amplified PONs could be estimated with the following equations, respectively;

$$
\begin{aligned}
& P_{D F B}=-10 \log \left(1-Q^{2} R^{2}\right), \\
& P_{R S O A}=-5 \log \left(1-16 Q^{2} R \frac{\kappa}{\pi B_{o} T}\right),
\end{aligned}
$$

where $Q$ is related to the signal-to-noise ratio (i.e., $Q=6$ at $\left.\mathrm{BER}=10^{-9}\right), R$ is the crosstalk-to-signal ratio, and $T$ is the bit duration of the modulated signal (inverse of data rate). The factor $K$ depends on the polarization states of signal and in-band crosstalk components, and $B_{o}$ is the equivalent optical bandwidth of the signal measured at the receiver. In our calculation, we used a $B_{o}$ of $34.2 \mathrm{GHz}$ for a BLS seeded RSOA source which was estimated from the optical spectrum of downstream signal measured after passing through two AWGs. In case of a DFB-LD, the calculated penalty agreed well with the measured penalty, as shown in Fig. 3(c). However, a small discrepancy was observed between the calculated and measured penalties with a BLS seeded RSOA source. Previously, we reported that the in-band crosstalk penalty with a BLS seeded optical source could be estimated with $\kappa=0.25$ in Eq. (2) [9]. This was because a depolarized ASE beam was used as a seeded source into a FP-LD or a RSOA. Therefore, we found that the discrepancy between the calculated and measured penalties was mainly due to the polarization dependent loss $(\sim 1 \mathrm{~dB})$ of our transmission links and polarization dependent gain $(<0.5 \mathrm{~dB})$ of our RSOA. Thus, we confirmed that the factor $K$ should be estimated properly by taking into account the polarization characteristics of the optical source and the 
transmission link in order to reduce the discrepancy between the calculated and measured in-band crosstalk penalties.

Previously, in order to increase the bidirectional gain of the optical amplifier, various optical components, such as optical bandpass filters [10] and interleavers [11] have been used for the suppression of unwanted reflected signal components. Therefore, as shown in Fig. 4(a), we also implemented the

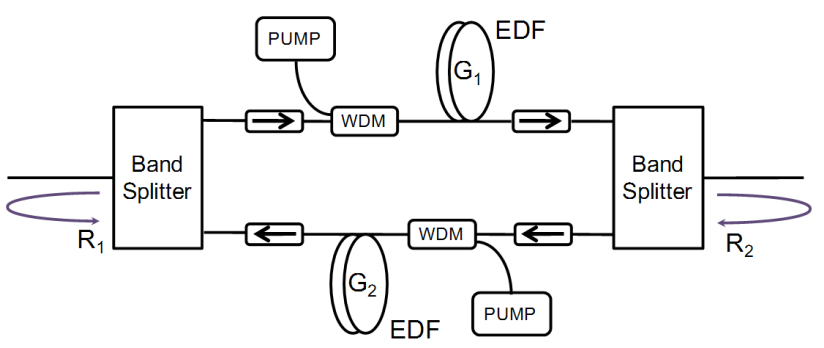

(a)

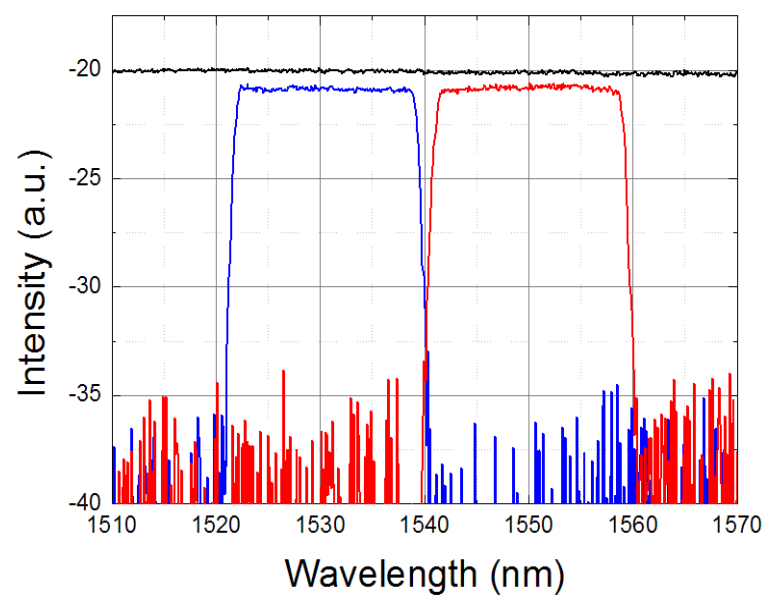

(b)

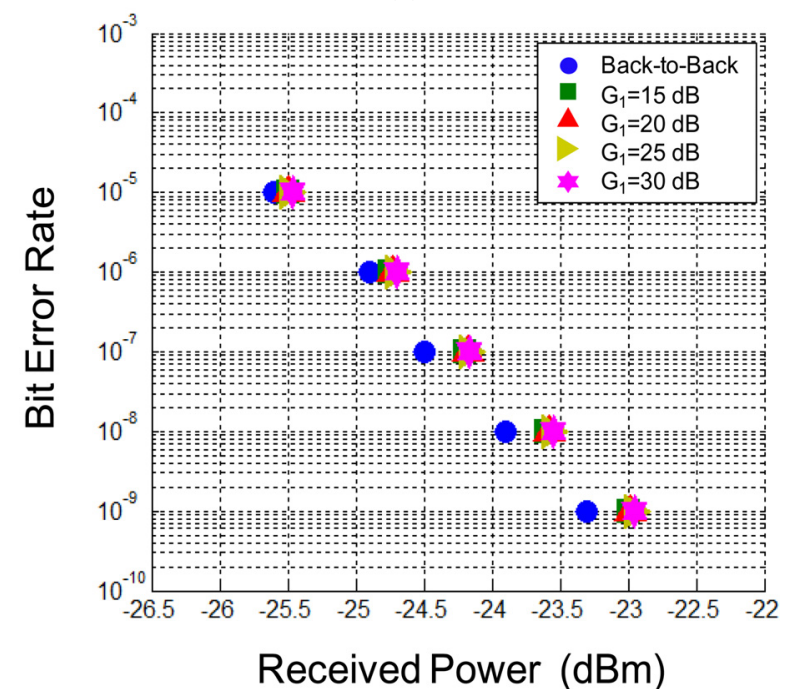

(c)

FIG. 4. (a) Bidirectional optical amplifier with two band splitters and two unidirectional EDFAs, (b) measured optical spectrum of a band splitter, and (c) measured BER curves with various gain $\left(\mathrm{G}_{1}\right)$ of optical amplifier for downstream signal. bidirectional optical amplifier with two band splitters to suppress the reflected signals in an amplified PON, where the separate wavelength bands were allocated to the downstream and upstream signals. Fig. 4(b) shows the measured transmission spectrum of our band splitter which provided two passbands; one (red band) for the downstream signals ranging from $1542 \mathrm{~nm}$ to $1558 \mathrm{~nm}$ and the other (blue band) for the upstream signals ranging from $1522 \mathrm{~nm}$ to $1538 \mathrm{~nm}$. We used the upstream signal of $1530.1 \mathrm{~nm}$ to match the passband (blue band) of band splitters in this measurement. As evident, the reflected $\left(\mathrm{R}_{2}\right)$ downstream signal could not be launched into and amplified with the unidirectional optical amplifier $\left(\mathrm{G}_{2}\right)$ of the upstream signal due to the filtering effect of the band splitter. Thus, the amount of in-band crosstalk generated within Fig. 4(a) could be reduced significantly, as compared to Fig. 3(a) and (b). In order to confirm the effective suppression of the unwanted reflected component, the bit-error-rate (BER) curves were measured as a function of amplifier gain $G_{1}$ for the downstream signal, as shown in Fig. 4(c). The amplifier gain $\mathrm{G}_{2}$ for the upstream signal was set to be $30 \mathrm{~dB}$ for all measurements. The back-to-back BER curve was also represented in Fig. 4(c), for reference. As can be seen in Fig. 4(c), a dispersion-induced penalty of $\sim 0.3 \mathrm{~dB}$ was observed after a $28-\mathrm{km}$ SMF transmission with a $1.25 \mathrm{~Gb} / \mathrm{s}$ downstream signal [12]. However, no in-band crosstalk induced penalties were observed even when the amplifier gain $\mathrm{G}_{1}$ was increased to as high as $30 \mathrm{~dB}$. This was because of the efficient suppression of the unwanted reflected signal by the band splitters. We believe that the amplifier gain could be increased to larger than $30 \mathrm{~dB}$ with a high power pump laser. From the results, we confirmed that the bidirectional optical amplifier with two band splitters was suited for use in the amplified PON which used the separated wavelength bands for downstream and upstream signals.

\section{SUMMARY}

We evaluated the bidirectional gains of optical amplifiers in an amplified PON with a BLS seeded RSOA source. Using a simple bidirectional amplifier without an optical isolator, we found that the bidirectional gain with a BLS seeded RSOA source could be increased to be as high as $27 \mathrm{~dB}$ with the reflectance coefficients of $-33 \mathrm{~dB}$ in both transmission fiber links. This was because a BLS seeded RSOA source was $\sim 10 \mathrm{~dB}$ more tolerant to the in-band crosstalk than a DFB-LD source. We also evaluated the gain of bidirectional optical amplifier with two band splitters for an amplified PON. From the results, we confirmed that the bidirectional optical amplifier with two band splitters could suppress the unwanted reflected signal efficiently, and then the bidirectional gain was increased to higher than $30 \mathrm{~dB}$ in the amplified PON which used the separated wavelength bands for the bidirectional signal transmission over a single fiber. 


\section{ACKNOWLEDGMENT}

This work was supported by Basic Science Research Program through the National Research Foundation of Korea (NRF) funded by the Ministry of Education, Science and Technology (MEST) (NRF-2008-331-D00375).

\section{REFERENCES}

1. R. P. Davey, P. Healey, I. Hope, P. Watkinson, D. B. Payne, O. Marmur, J. Ruhmann, and Y. Zuiderveld, "DWDM reach extension of a GPON to $135 \mathrm{~km}$," J. Lightwave Technol. 24, 29-31 (2006).

2. I. T. Monroy, R. Kjaer, B. Palsdottir, A. M. J. Koonen, and P. Jeppesen, "10 Gb/s bidirectional single fibre long reach PON link with distributed Raman amplification," in Proc. Eur. Conf. Optical Communication 2006 (Cannes, France, Sep. 2006), We3.P.166.

3. H. H. Lee, K. C. Reichmann, P. P. Iannone, X. Zhou, and B. Palsdottir, "A hybrid-amplified PON with 75-nm downstream band-with, $60 \mathrm{~km}$ reach, 1:64 split and multiple video services," in Proc. OFC/NFOEC 2007 (Anaheim, CA, USA, Mar. 2007), OWL2.

4. C. H. Kim, J. H. Lee, and K. Lee, "Analysis of maximum reach in WDM PON architecture based on distributed Raman amplification and pump recycling technique," Opt. Express 15, 14942-14947 (2007).
5. J. L. Gimlett, M. Z. Iqbal, N. K. Cheung, A. Righetti, F. Fontana, and G. Grasso, "Observation of equivalent Rayleigh scattering mirrors in lightwave systems with optical amplifier," IEEE Photon. Technol. Lett. 2, 211-213 (1990).

6. C.-H. Lee and S.-G. Mun, "WDM-PON based on wavelengthlocked Fabry-Perot LDs,” J. Opt. Soc. Korea 12, 326-336 (2008).

7. B. W. Kim, "RSOA-based wavelength-reuse gigabit WDMPON," J. Opt. Soc. Korea 12, 337-345 (2008).

8. M. O. van Deventer and O. J. Koning, "Bidirectional transmission using an erbium-doped fiber amplifier without optical isolators," IEEE Photon. Technol. Lett. 7, 1372-1374 (1995).

9. C. H. Kim, K. Lee, and S. B. Lee, "Effects of in-band crosstalk in wavelength-locked Fabry-Perot laser diode based WDM PONs," IEEE Photon. Technol. Lett. 21, 596-598 (2009).

10. F. Khaleghi, J. Li, M. Kavehrad, and H. Kim, "Increasing repeater span in high-speed bidirectional WDM transmission systems using a new bidirectional EDFA configuration," IEEE Photon. Technol. Lett. 8, 1252-1254 (1996).

11. M. F. Huang, J. Chen, K. M. Feng, C. C. Wei, C. Y. Lai, T. Y. Lin, and S. Chi, "210-km bidirectional transmission system with a novel four-port interleaver to facilitate unidirectional amplification," IEEE Photon. Technol. Lett. 18, 172-174 (2006).

12. C. H. Kim, J. H. Lee, D. K. Jung, Y.-G. Han, and S. B. Lee, "Performance comparison of directly-modulated, wavelengthlocked Fabry-Perot laser diode and EAM-modulated spectrumsliced ASE source for $1.25 \mathrm{~Gb} / \mathrm{s}$ WDM-PON," in Proc. OFC/NFOEC 2007 (Anaheim, CA, USA, Mar. 2007), JWA82. 